\title{
O papel do adipócito na inflamação e metabolismo do obeso
}

\author{
The role of adipocyte in the inflammation and metabolism of the obese \\ El papel del adipocitos em la inflamacíon y metabolismo del obeso
}

Recebido: 13/07/2021 | Revisado: 19/07/2021 | Aceito: 22/07/2021 | Publicado: 31/07/2021

\author{
Erica Fernanda Gomes de Sousa \\ ORCID: https://orcid.org/0000-0002-7047-6165 \\ Faculdade Estácio de Teresina, Brasil \\ E-mail: ericafernanda080898@gmail.com \\ Bruno Gabriel Santos Reis \\ ORCID: https://orcid.org/0000-0002-4913-3109 \\ Faculdade Estácio de Teresina, Brasil \\ E-mail: brunogab1999@gmail.com \\ Andrea Nunes Mendes de Brito \\ ORCID: https://orcid.org/0000-0002-8270-9018 \\ Faculdade Estácio de Teresina, Brasil \\ E-mail: drea.nunes@hotmail.com
}

\begin{abstract}
Resumo
Objetivo: Investigar o papel do adipócito na inflamação causada pela obesidade. Metodologia: Trata-se de uma revisão integrativa, realizada nas bases de dados LILACS, SCIELO e MEDLINE/PUBMED, no período de setembro de 2020 a maio de 2021, nos idiomas inglês, espanhol e português, publicados no intervalo de 2015 a maio de 2021. Foram utilizados os seguintes descritores: inflamação, metabolismo, adipócitos e obesidade, assim como suas versões em inglês. Resultados: O estado inflamatório produzido pelo estresse metabólico em decorrência da obesidade é diferente da inflamação clássica, o fator desencadeante é o excesso de nutrientes decorrente do crescimento e aumento do número de adipócitos, tornando-o um tecido disfuncional. As complicações metabólicas em um estado de obesidade podem ser agravadas por uma resposta inflamatória anormal e a mesma é conhecida por estar associada à formação de espécies reativas tóxicas de oxigênio. Conclusão: Conclui-se que os adipócitos exercem papel importante na inflamação e metabolismo do obeso, pois através dos adipócitos são produzidas as principais citocinas próinflamatórias, podendo acarretar em resistência à insulina, hipertensão, dentre outros problemas metabólicos.
\end{abstract}

Palavras-chave: Inflamação; Metabolismo; Adipócitos; Obesidade.

\begin{abstract}
Objective: To investigate the role of adipocytes in inflammation caused by obesity. Methodology: This is an integrative review, carried out in the LILACS, SCIELO and MEDLINE / PUBMED databases, in the period from September 2020 to May 2021, in English, Spanish and Portuguese language, published between 2015 and May 2021. The following descriptors were used: inflammation, metabolism, adipocytes and obesity, as well as their English versions. Results: The inflammatory state produced by metabolic stress due to obesity is different from classical inflammation, the triggering factor is the excess of nutrients resulting from growth and increase in the number of adipocytes, making it a dysfunctional tissue. Metabolic complications in a state of obesity can be aggravated by an abnormal inflammatory response and it is known to be associated with the formation of toxic reactive oxygen species. Conclusion: It is concluded that adipocytes play an important role in the inflammation and metabolism of the obese, because through adipocytes the main pro-inflammatory cytokines are produced, which can lead to insulin resistance, hypertension, among other metabolic problems.
\end{abstract}

Keywords: Inflammation; Metabolism; Adipocytes; Obesity.

\section{Resumes}

Objetivo: investigar el papel de los adipócitos em la inflamación causada por la obesidad. Metodología: Se trata de una revisión integradora, realizada en bases de datos LILACS, SCIELO e MEDLINE/PUBMED, em el período comprendido entre septiembre de 2020 y mayo de 2021, em inglés, español y portugués, publicado en el período de 2015 a mayo de 2021. Se utilizaron los seguientes descriptores: inflamación, metabolismo, adipocitos y obesidad, así como sus versiones em inglés. Resultados: El estado inflamatorio producido por el estrés metabólico debido a la obesidad es diferente al de la inflamación clássica, o factor desencadenante es el excesso de nutrientes resultantedel crecimiento y aumento del número de adipocitos, convirtiéndolo em un tejido disfuncional. Las complicaciones metabólicas en un estado de obesidade pueden agravarse por una respuesta inflamatória anormal y se sabe que está asociado con la formación de especies tóxicas de oxígeno reactivo. Conclusión: Se concluye que los adipocitos juegan un papel importante en la inflamación y metabolismo de los obesos, porque a traves de los adipocitos se producen las principales citocinas proinflamatorias, puede provocar resistencia a la insulina, hipertensión, entre otros problemas metabólicos.

Palabras clave: Inflamación; Metabolismo; Adipocitos; Obesidad. 


\section{Introdução}

A obesidade é definida pela Organização Mundial de Saúde (OMS) como acúmulo anormal ou excessivo de gordura corporal, suficiente para que altere ou prejudique as funções fisiológicas do organismo (Santos, 2017). Uma das causas fundamentais da obesidade segundo Francisqueti 2015, é a relação do desequilíbrio energético entre as calorias consumidas e as gastas. Atualmente, é considerada um problema de saúde pública por estar associada ao alto risco de doenças cardiovasculares, dislipidemias, diabetes tipo II , além de possíveis cânceres.

Segundo o Ministério da Saúde (2019), o número de obesos no Brasil entre os anos de 2006 a 2018 aumentou em 67,8 $\%$ e apontam que o crescimento da mesma foi maior entre os adultos de 25 a 34 anos e 35 a 44 anos de idade, com índices de crescimento em $84,2 \%$ e $81,1 \%$ respectivamente. Com isso, a prevalência da obesidade voltou a crescer no país. Segundo dados da Vigilância de Fatores de Risco e Proteção para Doenças Crônicas por Inquérito Telefônico - VIGITEL (2018), em 2006 o índice de prevalência acabou saindo de 11,8\% para 19,8\% no ano de 2018.

Esta condição pode estar relacionada a várias anormalidades metabólicas, doenças crônicas, possível agravamento de doenças cardiovasculares, além de, geralmente, ser acompanhada por um certo grau de inflamação, denominada inflamação crônica de baixa intensidade (Magnuson et al., 2018).

Nessa condição de inflamação crônica, o tecido adiposo (TA) se torna responsável por aumentar a secreção de mediadores inflamatórios, como TNF- $\alpha$ (fator de necrose tumoral alfa) e IL-6 (interleucina-6) (Nascimento, 2021), por meio do fator de transcrição nuclear Kappa (NF-kappaB), considerado o principal regulador da expressão dessas moléculas e auxilia a patogênese de várias doenças, no qual geralmente está relacionada à disfunção dos adipócitos (Cozzolino \& Cominetti, 2016).

Sendo a principal reserva de energia do corpo humano, os adipócitos presentes no TA, são as únicas células que armazenam lipídios na forma de triacilgliceróis (TAG) no citoplasma, além de serem capazes de sintetizar ácidos graxos (lipogênese) e estocar TAG em períodos em que a oferta de energia é abundante e mobilizá-los pela lipólise quando há déficit calórico (Cozzolino \& Cominetti, 2016). Apesar da sua grande importância, em excesso (característico da obesidade), pode acarretar consequências a qualidade de vida (Soares et al., 2020).

Embora o mecanismo subjacente ainda não seja totalmente compreendido, o aumento no tamanho dos adipócitos e a incapacidade de armazenar TAG em condições de superalimentação parecem ser críticos para as mudanças nos padrões metabólicos disfuncionais, pois estão relacionadas às vias de inflamação e apoptose (morte celular programada), como um recurso para a secreção de adipocinas pró-inflamatórias (Francisqueti et al., 2016).

Visando colaborar para o entendimento da patologia, bem como identificar meios quem favoreçam seu tratamento de forma segura e eficaz, a pesquisa tem como objetivo investigar as ações do adipócito no metabolismo e na inflamação causada pela obesidade.

\section{Metodologia}

O presente estudo, trata-se de uma revisão integrativa que consiste na construção de uma análise ampla da literatura, contribuindo para reflexões sobre a realização de futuros estudos.

Para a construção da revisão integrativa foi preciso percorrer seis etapas distintas: 1) identificação do tema e seleção da hipótese ou questão de pesquisa para elaboração da revisão integrativa; 2) estabelecimento de critérios para inclusão e exclusão de estudos; 3) definição das informações a serem extraídas dos estudos selecionados; 4) avaliação dos estudos incluídos na revisão integrativa; 5) interpretação dos resultados; 6) apresentação da revisão/síntese do conhecimento (Mendes et al., 2008). 
Para a construção da pergunta norteadora "Qual o papel dos adipócitos na inflamação e metabolismo do obeso?" foi utilizada a estratégia PICo, no qual cada acrônimo representa um componente da questão, "P" de paciente ou problema (Inflamação e metabolismo); "I" de intervenção ou indicador (Papel dos adipócitos); "Co" de contexto (Obesidade). Essa estratégia permite localizar de forma específica informações científicas (evidências) sobre as questões que se deseja investigar (Veronese et al., 2015).

A pesquisa foi realizada nas bases de dados Literatura latino-americana e do caribe em ciências da saúde (LILACS), Scientific Electronic Library Online (SCIELO) e Medical Literature Analysis and Retrieval System Online (MEDLINE/PUBMED), no período de setembro de 2020 a maio de 2021, utilizando os seguintes descritores: inflamação, metabolismo, adipócitos e obesidade, assim como as seguintes combinações: inflamação/inflammation OR metabolismo/metabolism AND adipócitos/adipocytes AND obesidade/obesity.

Os critérios de elegibilidade utilizados foram artigos originais e de revisão gratuitos disponíveis na íntegra, nos idiomas inglês, espanhol e português, publicados no intervalo de 2015 a maio de 2021 que aborde o tema proposto. Os artigos não condizentes com a temática de estudo, relatos de caso, dissertações, bem como aqueles que datavam antes de 2015 e publicações duplicadas nas diferentes bases de dados foram excluídos.

Ao ser realizada a estratégia de busca foram identificados 1053 estudos, dos quais 1037 foram descartados por não atenderem aos critérios de elegibilidade, após a realização das etapas da revisão. Dessa maneira, 16 artigos foram inclusos na revisão, conforme mostra a Figura 1.

Figura 1 - Fluxograma da seleção de artigos.

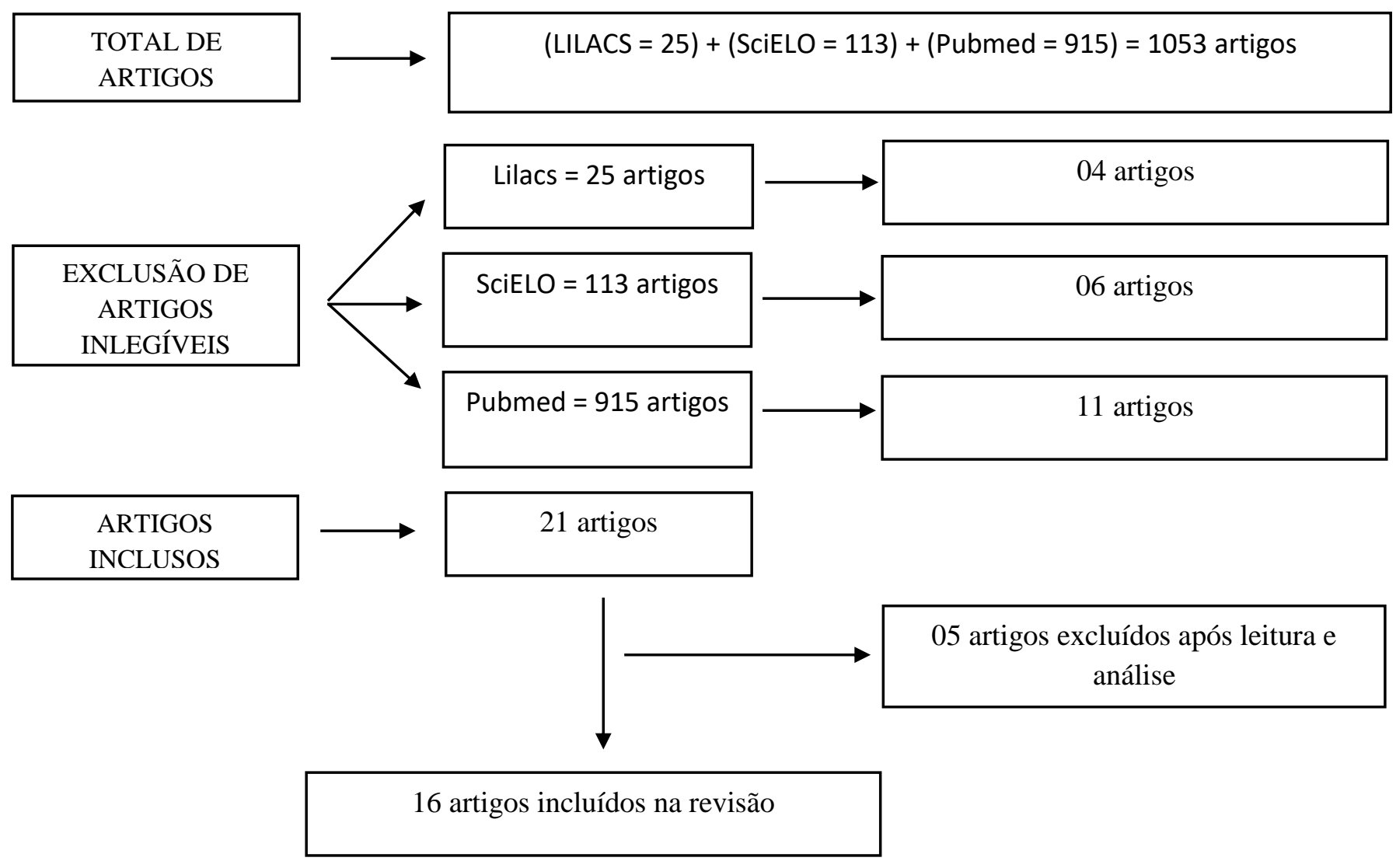

Fonte: Autores (2021). 
$\mathrm{Na}$ etapa de avaliação crítica foi realizada uma leitura meticulosa e analítica dos títulos e resumos dos artigos. Após a conclusão desta etapa, os artigos selecionados foram lidos na íntegra. Este procedimento foi realizado por dois pesquisadores simultaneamente e de forma independente, levando em consideração os critérios de elegibilidade e exclusão pré-definidos. Os trabalhos para os quais houve discordância foram analisados em reunião com os autores para avaliação e consenso sobre a inclusão na revisão. A extração dos dados foi realizada por meio de um protocolo elaborado pelos pesquisadores, no qual foram incluídos os seguintes dados: autor/ano, objetivo, metodologia e principais resultados que foram apresentados no formato de quadro.

\section{Resultados e Discussão}

Ao analisar os estudos encontrados, nota-se que a inflamação em decorrência da obesidade se difere da inflamação popularmente conhecida, caracterizada por odor, dor e aparência inchada, pois nessa situação, segundo Francisqueti (2015), o que desencadeia o processo inflamatório é o excesso de nutrientes que é observado em decorrência do crescimento e aumento do número de adipócitos. Tal processo modifica a funcionalidade e mecanismos do TA, tornando-o um tecido desregular. A relação entre os estudos e autores encontrados se apresenta no Quadro 1 a seguir.

Quadro 1 - Caracterização dos estudos.

\begin{tabular}{|c|c|c|c|}
\hline Autor/Ano & Objetivo & Tipo de estudo/amostras & Principais Resultados \\
\hline Júnior, 2017 & $\begin{array}{l}\text { Elaborar explicação que } \\
\text { relaciona a obesidade com o } \\
\text { Diabetes Tipo } 2 \text { a partir dos } \\
\text { elementos: adipocinas, TNF- } \\
\alpha, \quad \text { IL-6, resistina e } \\
\text { adiponectina. }\end{array}$ & $\begin{array}{l}\text { Trata-se de uma revisão de } \\
\text { literatura. As amostras foram } \\
\text { estudos publicados em revistas } \\
\text { indexadas; referências } \\
\text { bibliográficas constantes nos } \\
\text { estudos selecionados; adipocinas } \\
\text { abordadas nestes estudos; } \\
\text { obesidade e diabetes tipo } 2 \text {. }\end{array}$ & $\begin{array}{l}\text { Com a elevação da massa do adipócito, } \\
\text { ocorrem alterações histológicas e bioquímicas, } \\
\text { além da migração de macrófagos que estimula a } \\
\text { expressão, síntese e produção de fatores } \\
\text { inflamatórios, as adipocinas pró-inflamatórias. } \\
\text { A ação conjunta destas adipocinas promove } \\
\text { sindrome metabolica, como hipertensão arterial } \\
\text { e diabetes tipo } 2 \text {. }\end{array}$ \\
\hline Silva et al., 2019 & $\begin{array}{l}\text { Relacionar o papel das } \\
\text { adipocinas na obesidade com } \\
\text { suas funções endócrinas e } \\
\text { inflamatórias }\end{array}$ & $\begin{array}{l}\text { Trata-se de uma revisão } \\
\text { bibliografica. Os instrumentos } \\
\text { utilizados foram artigos, livros e } \\
\text { publicações científicas nacionais e } \\
\text { internacionais, relacionado entre } \\
\text { as principais adipocinas. }\end{array}$ & $\begin{array}{l}\text { Essas adipocinas em desequilíbrio promovem } \\
\text { grande impacto em diversas funções corporais, } \\
\text { alterando a ingesta alimentar, imunidade, ações } \\
\text { metabolicas da insulina, angiogênese, pressão } \\
\text { arterial, metabolismo lipídico e equilibrio } \\
\text { energetico. }\end{array}$ \\
\hline $\begin{array}{l}\text { Francisqueti } \text { et al., } \\
\qquad 2015\end{array}$ & $\begin{array}{lr}\text { Abordar os } & \text { aspectos } \\
\text { inflamatórios, adipocinas e os } \\
\text { principais } & \text { mecanismos } \\
\text { envolvidos } & \text { no } \\
\text { desenvolvimento } & \text { das } \\
\text { complicações } & \text { metabólicas } \\
\text { relacionadas à obesidade. }\end{array}$ & $\begin{array}{l}\text { Trata-se de uma revisão da } \\
\text { literatura e foram utiizados artigos } \\
\text { levantados por meio de pesquisa } \\
\text { em diferentes bases de dados. }\end{array}$ & $\begin{array}{l}\text { A inflamação pode ser desencadeada por } \\
\text { diferentes mecanismos moleculares e excesso } \\
\text { de nutrientes, o qual se manifesta com aumento } \\
\text { do tamanho e número dos adipócitos, chegando } \\
\text { a um quadro de síndrome metabólica que } \\
\text { compromete o sistema cardiovascular. A } \\
\text { secreção de citocinas inflamatórias no interior } \\
\text { das células as quais prejudicam as vias de } \\
\text { sinalização da insulina, podem levar à } \\
\text { deterioração da resposta do tecido ao hormônio. }\end{array}$ \\
\hline Dludla et al., 2018 & \begin{tabular}{lr} 
Revisar os & \multicolumn{2}{r}{ mecanismos } \\
envolvidos nos & efeitos \\
fisiopatológicos & da \\
inflamação e do estresse \\
oxidativo em obesos.
\end{tabular} & $\begin{array}{l}\text { Trata-se de uma revisão da } \\
\text { literatura. Foram utilizados } \\
\text { evidencias disponiveis que relatam } \\
\text { as propiedades antiobesidade com } \\
\text { ênfase em seu efeito modulador } \\
\text { nos mecanismos moleculares } \\
\text { envolvidos na sinalização, } \\
\text { inflamação e estresse oxidativo da } \\
\text { insulina. }\end{array}$ & $\begin{array}{l}\text { O desequilíbrio entre a expansão do tecido } \\
\text { adiposo e sua vascularização resulta em } \\
\text { hipóxia, que promove a inflamação dos } \\
\text { adipócitos além de uma infiltração de } \\
\text { macrófagos inflamatórios nos mesmos. A } \\
\text { inflamação e estresse oxidativo são alguns dos } \\
\text { principais fatores causais implicados no } \\
\text { agravamento das perturbações relacionadas a } \\
\text { obesidade. }\end{array}$ \\
\hline
\end{tabular}




\begin{tabular}{|c|c|c|}
\hline Lacerda et al., 2016 & $\begin{array}{l}\text { Descrever os efeitos } \\
\text { metabolicos de diferentes } \\
\text { adipocinas a partir da nova } \\
\text { perspectiva do tecido adiposo } \\
\text { como orgão endocrino. }\end{array}$ & $\begin{array}{l}\text { Trata-se de uma revisão literária. } \\
\text { O material obtido foram estudos } \\
\text { originais com limite temporal de } \\
2010 \text { no idioma inglês. }\end{array}$ \\
\hline & $\begin{array}{ll}\text { Estudar as principais } \\
\text { adipocinas envolvidas no } \\
\text { estado inflamatório dos } \\
\text { adipócitos na obesidade. }\end{array}$ & $\begin{array}{l}\text { Trata-se de uma revisão literária e } \\
\text { foram utilizados artigos levantados } \\
\text { por meio de pesquisa nos bancos } \\
\text { de dados da literatura. }\end{array}$ \\
\hline
\end{tabular}

Santos, 2017 $\begin{array}{lll}\text { estado inflamatório dos } & \text { por meio de pesquisa } \\ \text { adipócitos na obesidade. } & & \text { de dados da literatura. }\end{array}$

Os adipócitos secretam uma variedade de substâncias nomeadas adipocinas, que por sua vez interferem no processo fisiológico de doenças metabólicas e cardiovasculares, além de um papel importante em suas fisiopatologias e no processo inflamatório.

As adipocinas secretadas nos adipócitos são múltiplos peptídeos bioativos que em concentrações elevadas promovem grande impacto em diversas funções corporais. No geral este quadro predispõe a intensa produção de substâncias maléficas ao organismo e estabelecimento de resistência insulínica, induzindo reações inflamatórias locais e sistêmicas, predispondo ao aparecimento de fatores associados à síndromes metabólicas.

\begin{abstract}
Analisar os mecanismos de ação de moléculas antiinflamatórias e compostos sobre adipócitos.
\end{abstract}

Nascimento et al, 2021

Liechocki, 2018

Entender a participação dos diferentes depósitos de TA branco no quadro inflamatório associado à obesidade.
Trata-se de um protocolo para revisão sistemática e meta-analise. Os materiais utilizados são artigos levantados em bases de dados, além de estudos experimentais realizados em ratos e camundongos com grupo controle que descreva o tratamento com antiinflamatórios em qualquer frequência, horário e dose

Trata-se de um estudo qualitativo exploratório. As amostras utilizadas foram, indivíduos com IMC acima de 35, portadores ou não de hipertensão, diabetes mellitus tipo II e/ou dislipidemias e que não possuíam quaisquer outras moléstias não associadas à obesidade.

Compreensão dos Trata-se de uma revisão da mecanismos regulatórios da função dos macrófagos do TA (ATM) no processo inflamatório da obesidade.

litaratura. As amostras são artigos levantados em base de dados.
Os desfechos incluem a identificação do efeito do tratamento com antiinflamatórios no TA, redução de marcadores inflamatórios como gordura corporal, expressão gênica e dosagens de citocinas, aumento dos níveis de adiponectina, redução da expressão da enzima 5-lipoxigenase (5-LOX), melhor sensibilidade à insulina e redução da PCR.

O TA subcutâneo apresentou maior conteúdo de diversas citocinas pró-inflamatórias. Os dados levam a crer que os três depósitos de TA abdominal contribuem sinergicamente para o estabelecimento do perfil de citocinas séricas e que tais características podem ser decisivas para o estabelecimento do quadro de obesidade mórbida destes pacientes.

Os ATMs desempenham um papel fundamental na patogênese da inflamação causada pela obesidade e nas complicações metabólicas. A liberação de produtos secretores (citocinas e RNAs extracelulares) é uma forma crítica que os ATMs aplicam para regular outras células, bem como para coordenar as respostas inflamatórias.

Examinar a expressão de IL6 Trata-se de um estudo pelo TA durante a resposta à experimental. As amostras obesidade.

Han et al, 2020 utilizadas foram camundongos

Investigar o fator extrínseco do TA a inflamação causada

Magnuson et al, pela obesidade e o papel do retrocruzados para a cepa C57BL / 6J (10 gerações) e alojados em uma instalação livre de patógenos específica.

Mostrou que a fonte de expressão da citocina pode determinar a resposta de sinalização inflamatória. Em contraste, os adipócitos podem secretar IL6 em resposta à obesidade e aumentar o acúmulo de ATM. Essa plasticidade mecanística permite que a IL6 promova uma resposta biológica apropriada dentro de um contexto fisiológico específico.

$\begin{array}{lll}\text { Trata-se de um estudo } & \text { A desregulação da função endócrina do TA, } \\ \text { experimental, no qual } & \text { citocinas pró-inflamatórias e citocinas } \\ \text { camundongos machos do Jackson } & \text { deletérias estão implicadas na fisiopatologia de } \\ \text { Laboratory foram utilizados como } & \text { várias doenças. Os efeitos deletérios da } \\ \text { amostra. } & \text { expansão excessiva do depósito visceral, em } \\ & \text { parte, são devidos ao aumento da produção e }\end{array}$




\begin{tabular}{|c|c|c|c|}
\hline & & & $\begin{array}{l}\text { liberação de citocinas pró-inflamatórias. A } \\
\text { liberação de citocinas do TA se origina tanto } \\
\text { dos adipócitos quanto das células imunes } \\
\text { contidas no mesmo. }\end{array}$ \\
\hline Carmona et al, 2017 & $\begin{array}{l}\text { Apresentar o estado atual da } \\
\text { fisiopatologia da obesidade } \\
\text { de forma simples e didática, } \\
\text { com foco no TA. }\end{array}$ & $\begin{array}{lcr}\text { Trata-se de } & \text { uma } & \text { revisão } \\
\text { bibliográfica. } & \text { Os } & \text { materiais } \\
\text { utilizados } & \text { foram } & \text { artigos } \\
\text { levantados. } & & \end{array}$ & $\begin{array}{l}\text { As células imunes do TA têm a capacidade de } \\
\text { secretar fatores inflamatórios, que se torna } \\
\text { essencial para determinar o papel que } \\
\text { alterações dos adipócitos têm no conceito } \\
\text { metabólico, passando de um perfil } \\
\text { antiinflamatório a inflamatório. Na obesidade a } \\
\text { maioria das citocinas com perfil pró- } \\
\text { inflamatório são emitidas por macrófagos M1 } \\
\text { ou "classicamente ativados" do TA. }\end{array}$ \\
\hline Longo et al, 2019 & $\begin{array}{l}\text { Resumir o conhecimento } \\
\text { atual sobre a disfunção do } \\
\text { TAB na obesidade e suas } \\
\text { anormalidades metabólicas } \\
\text { associadas. }\end{array}$ & $\begin{array}{l}\text { Trata-se de uma revisão } \\
\text { bibliográfica. Os materiais de } \\
\text { estudos foram artigos levantados } \\
\text { para comparação e discussão. }\end{array}$ & $\begin{array}{l}\text { A capacidade de expansão limitada do TA } \\
\text { subcutâneo leva a uma expansão inadequada } \\
\text { das células adiposas com inflamação local e } \\
\text { fenótipo de resistência à insulina. A hipertrofia } \\
\text { dos adipócitos também resulta em deficiência } \\
\text { da vasculatura e hipóxia local do TA. A hipóxia } \\
\text { é um significativo gatilho para a indução da } \\
\text { inflamação dos adipócitos. }\end{array}$ \\
\hline
\end{tabular}

Fonte: Autores (2021).

$\mathrm{O}$ adipócito inflamado promove a síntese e o fluxo de fatores inflamatórios, como as adipocinas, as quais tem envolvimento e contribuição para hipertensão e baixa atividade insulínica (Júnior, 2017). Citocinas, quimiocinas e moléculas quimiotáticas como MCP-1 e IL-8 que são liberadas pelos adipócitos, são responsáveis por recrutar essas células de expansão e provocar um processo inflamatório.

Um estudo foi capaz de identificar 34 quimiocinas secretadas nos adipócitos, onde algumas são capazes de atrair monócitos do sangue e desenvolverem-se em macrófagos ao adentrar nos adipócitos, fazendo aumentar a produção de citocinas inflamatórias, identificando também que a expansão não controlada de TA se associa ao metabolismo lipídico disfuncional (Dludla, 2018). De acordo com seu tipo essas adipocinas podem desempenhar papeis distintos, como indução à saciedade (leptina) e a regulação da resistência insulínica (resistina) (Soares et al, 2020).

A obesidade caracterizada como uma inflamação crônica de baixo grau segundo Magnuson et al., 2018; Francisqueti et al., 2015 e Russo \& Lumeng, 2018 proporciona aumento significativo na massa dos adipócitos, principalmente o visceral, que ocorre alterações histológicas e bioquímicas características de tecido inflamado (Júnior, 2017). Atualmente são conhecidas cerca de 50 diferentes moléculas produzidas e/ou secretadas pelo TA (Silva et al, 2019).

O estado inflamatório na obesidade também é caracterizado por manifestações de hipertrofia e hiperplasia dos adipócitos (Francisqueti et al., 2015). O adipócito é a principal célula presente no TA, é especializado em armazenar energia na forma de triglicerídeos em seus corpos lipídicos (sendo a única célula que não pode sofrer lipotoxicidade) e liberá-los quando existir necessidade energética. Desde a sua descoberta como célula endócrina, é compreendido que ele desempenha um papel ativo tanto no balanço energético como em inúmeros processos metabólicos e fisiológicos (Carmona et al., 2017). O adipócito inflamado promove a expressão, secreção e síntese de fatores inflamatórios (Júnior, 2017).

O grau de inflamação característico da obesidade é caracterizado pelo alto índice de produção de adipocinas inflamatórias através dos adipócitos, estes fazem surtir efeitos endócrinos, parácrinos e autócrinos provocando uma inflamação metabólica e ocasionando o aumento do número de células inflamatórias (Francisqueti et al., 2015). É apontado que a inflamação associada a obesidade é incitada e exacerbada pela deposição excessiva de TA desregulado. Está desregulação da função endócrina, além de citocinas deletérias e pró-inflamatórias estão implicadas na fisiopatologia de várias doenças. O 
acúmulo de tecido adiposo visceral (TAV), em particular, está altamente associado a perfis metabólicos adversos, especificamente sua pró-inflamação causa vazamento gastrointestinal e a inflamação do intestino leva ao acúmulo de TA, os dois fatores juntos exacerbam a pró-inflamação sistêmica (Magnuson et al., 2018).

Essas adipocinas em desequilíbrio promovem grande impacto em diversas funções corporais, alterando a ingesta alimentar, sensibilidade insulínica, imunidade, angiogênese, pressão arterial, metabolismo lipídico e o balanceamento energético (Silva et al., 2019). Os macrófagos têm sido considerados uma fonte significativa de citocinas inflamatórias (podem determinar a resposta de sinalização inflamatória), cuja produção pode ser modulada de forma variável pela atividade da NADPH oxidase 2 durante a progressão da obesidade (Russo \& Lumeng, 2018; Han et al., 2020).

$\mathrm{Na}$ obesidade, podem ocorrer alterações metabólicas que são agravadas por uma resposta inflamatória anormal, crescimento de espécies reativas de oxigênio e subsequente geração de estresse oxidativo. Como discutido por Francisqueti et al, 2015 e corroborando Dludla et al, 2018, os adipócitos de indivíduos com obesidade possuem um perfil de citocinas alterado, como secreção e expressão de TNF- $\alpha$ e IL-6.

Sintetizado pelo tecido muscular, fração vascular estromal e por adipócitos, o TNF- $\alpha$ tem função pró-inflamatória, além de está implicado na citotoxicidade, resistência insulínica, apoptose e síntese de outras citocinas, como a interleucina-1 (IL-1) e a IL-6 (Júnior, 2017; Lacerda et al., 2016).

Os adipócitos tem um papel importante no que diz respeito a regulação do acúmulo gorduroso no TA (Lacerda et al., 2016). O TNF- $\alpha$ tem sido associado à patogênese da obesidade e diabetes tipo 2 , sendo indicado como principal fator responsável da resistência à insulina, sua expressão e liberação está relacionada à infiltração de macrófagos, portanto, ligado ao índice de massa corpórea (IMC) e obesidade (Júnior, 2017).

A leptina, é considerada adipocina e tem capacidade de modular a produção de TNF- $\alpha$, esta por feedback positivo, estimula a expressão do mesmo, de IL-6 e suprime a expressão de adiponectina, promovendo elevação das adipocinas próinflamatórias e atenuação da anti-inflamatória (Júnior, 2017; Lacerda et al.,2016).

Pode-se inferir que as adipocinas TNF- $\alpha$, resistina e IL-6 promovem a resistência insulínica por redução na fosforilação dos receptores de insulina. A literatura refere-se que, dentre as adipocinas expressas pelos adipócitos, a adiponectina é um regulador chave da captação à insulina e provoca efeito antagônico a estas adipocinas pró-inflamatórias (Júnior, 2017).

Em virtude da ação do TNF- $\alpha$, em indivíduos obesos e diabéticos do tipo 2, os níveis séricos de adiponectina são reduzidos. Estudos indicam que esta ação do TNF- $\alpha$ em promover hipoadiponectinemia esteja relacionada às causas ambientais (obesidade promovendo elevação de TNF- $\alpha$ ). Entretanto, a hipoadiponectinemia pode ser induzida também por uma alteração gênica (Júnior, 2017).

A incitação crônica na produção de citocinas pró-inflamatórias dentro das células que sofrem ação da insulina pode prejudicar a resposta do tecido ao hormônio. Corroborando com essa ideia Francisqueti et al., 2015, evidenciam altos níveis de citocinas pró-inflamatórias no soro e no TA de indivíduos e animais obesos com resistência insulínica. O TNF- $\alpha$ inibe a diferenciação dos adipócitos, e reduz sua capacidade de armazenar lipídios, condição esta que pode acentuar a hiperlipidemia e a lipotoxicidade nos demais órgãos em indivíduos obesos (Santos, 2017).

A IL-6 possui efeito pró-inflamatório e ação no metabolismo de carboidratos e lipídeos (Lacerda et al., 2016). Os adipócitos podem secretar IL-6 em resposta à obesidade (Han et al., 2020), expressa, juntamente com seu receptor (IL-6R) pelo adipócito e matrix do TA, principalmente o visceral (do fígado), influencia o metabolismo de lipídeos, através da inibição da lipase lipoproteica e da indução de lipólise (Júnior, 2017; Santos, 2017).

Sua expressão e concentrações séricas correlacionam-se com a obesidade, resistência à insulina e diabetes tipo 2 . A IL-6 em conjunto com o TNF- $\alpha$ elevam a liberação de AGLs e glicerol pelos adipócitos, aumenta a secreção de triglicerídeos 
pelo fígado e favorece a supressão da expressão de adiponectina; efeito sobre a resistência à insulina e o diabetes tipo 2 (Júnior, 2017).

A expressão elevada de IL-6 pode estar relacionada à supressão de leptina e estimulação da produção de Proteína C Reativa, redução da expressão de Insulin Receptor Substrate 1 e GLUT-4 nos tecidos muscular e hepático. Indivíduos portadores de patologias cardiovasculares mostraram elevados teores de IL-6. Segundo a pesquisa bibliográfica, a IL-6 pode constituir fator de interligação entre a aterosclerose e o processo inflamatório devido a associação negativa entre IL-6 e HDL-c e a forte associação positiva entre essa citocina e mediadores inflamatórios (Lacerda et al., 2016).

A leptina, a adiponectina e a resistina são adipocinas que também participam da resistência insulínica e do processo inflamatório em pessoas obesas. A leptina aumenta de forma expressiva com a gordura corporal, promovendo uma ligação funcional entre obesidade e doenças cardiovasculares (Francisqueti et al., 2015). Mediadores pró-inflamatórios e obesidade parecem ser os principais fatores responsáveis pelo aumento da produção de leptina (Santos, 2017).

A obesidade tem sido associada a um distúrbio no perfil secretório tanto do TA quanto do adipócito, observando-se uma alteração na relação leptina/adiponectina. Portanto, em um contexto de lipo-inflamação, observa-se o aumento dos níveis séricos de leptina acompanhado de diminuição da adiponectina que não corresponde aos níveis de TA. Além disso, o papel imunomodulador desempenhado pela leptina, e o papel anti-inflamatório e sensibilizador da insulina no nível sistêmico da adiponectina resulta em um perfil secretor que pode explicar em parte as anormalidades metabólicas correlacionadas a obesidade, como uma condição que carrega inflamação de baixo grau (Carmona et al., 2017).

A adiponectina possui ação anti-inflamatória e antiaterogênica, atua como protetor cardiovascular e melhora a sensibilidade à insulina. A ação anti-inflamatória ocorre por meio da supressão do NF-kappaB em macrófagos e monócitos, suspendendo processos induzidos pelo TNF- $\alpha$ (inflamação/adesão de monócitos) (Francisqueti et al., 2015).

Os efeitos metabólicos da adiponectina no fígado eleva a sensibilidade à insulina, restringe o influxo de ácidos graxos não esterificados, eleva a oxidação dos ácidos graxos e reduz o fluxo de glicose (Júnior, 2017). Pesquisas apontam que o TNFa e a IL-6 são potentes inibidores da expressão e excreção de adiponectina no TA humano e cultura de células (Francisqueti et al., 2015). Em indivíduos que apresentaram redução dos teores circulantes da adiponectina, foram observadas disfunções metabólicas associadas. Uma parte significativa dos indivíduos manifesta diabetes, hipertensão arterial, dislipidemia e aterosclerose, sugerindo a associação entre hipoadiponectinemia e o estabelecimento da síndrome metabólica (Lacerda et al., 2016).

A resistina também é uma adipocina pró-inflamatória, expressa nos adipócitos e macrófagos, e está intimamente relacionada ao IMC e a obesidade. Quando analisado a concentração de resistina em relação a massa do adipócito, observa-se que a sua expressão e seus níveis circulantes são mais acentuados em obesos quando comparados a indivíduos eutróficos (Júnior, 2017).

Segundo Santos, 2017, ao contrário da adiponectina, a resistina quando aumentada, pode induzir a produção de moléculas inflamatórias sobre as células do endotélio vascular, predispondo a doenças cardiovasculares em humanos. Além de ser um marcador inflamatório de grande potencial pró-inflamatório em humanos, está associada a resistência insulínica e síndrome metabólica (Francisqueti et al., 2015). Assim, Lacerda et al., 2016 especulam que a resistina possa representar o elo entre inflamação e sinalização metabólica.

Portanto, de acordo com Carmona et al., 2017, o estado de inflamação tecidual conhecido como lipo-inflamação, libera na circulação fatores inflamatórios que podem viajar para outros tecidos, gerando alterações nos mesmos e dando origem a um quadro inflamatório sistêmico de baixo grau. Ao lado de alterar a angiogênese, representa um cenário de hipóxia e alteração da matriz extracelular (fibrose) inflamatória agravando ainda mais o quadro da mesma. 


\section{Conclusão}

A partir dos achados da presente pesquisa, conclui-se que os adipócitos exercem papel importante no metabolismo e na inflamação provocada pela obesidade, pois é através dos adipócitos que são produzidas as principais citocinas próinflamatórias, podendo acarretar em resistência insulínica, hipertensão arterial sistêmica, dentre outros problemas metabólicos.

Portanto, faz-se necessário controlar o estado de inflamação em que o TA entra em decorrência da hipertrofia e hiperplasia dos adipócitos, sendo isso possível por meio de reeducação alimentar, atividade física regular e decorrente perda de peso. Tais mudanças de hábitos de vida exercem influência positiva nas variáveis em questão, explanadas e discutidas pelo presente estudo.

Dessa maneira, espera-se que os resultados do referido estudo sirvam de apoio a comunidade cientifica, acadêmicos e profissionais que buscam o entendimento sobre as ações dos adipócitos em decorrência do processo inflamatório da obesidade e por fim, ressaltamos ainda, a necessidade de estudos mais profundos por se tratar de um assunto complexo e repleto de variáveis.

\section{Referências}

Cozzolino, S. M. F., \& Cominetti, C. (2016). Bases bioquímicas e fisiológicas da nutrição. Manole.

Dludla, P, D., Nkambule, B, B., Jack, B., Mkandla, Z., Mutize, T., Silvestri, S., Orlando, P., Tiano, L., Louw, J., \& Mazibuko-Mbeje, S. (2018). Inflammation and Oxidative Stress in an Obese State and the Protective Effects of Gallic Acid. Nutrients, 11(1), 23. https://doi.org/10.3390/nu11010023

Francisqueti, F. V., Nascimento, A. F., \& Corrêa, C. R. (2015). Obesidade, inflamação e complicações metabólicas. Nutrire, 40 (1), 81-89. http://sban.cloudpainel.com.br/files/revistas_publicacoes/452.pdf

Han, M. S., White, A., Perry, R. J., Camporez, J.-P., Hidalgo, J., Shulman, G. I., \& Davis, R. J. (2020). Regulation of adipose tissue inflammation by interleukin 6. Proceedings of the National Academy of Sciences, 117(6), 2751-2760. https://doi.org/10.1073/pnas.1920004117

Júnior, A. J. S. (2017). Adipocinas: a relação endócrina entre obesidade e diabetes tipo II. RBONE - Revista Brasileira de Obesidade, Nutrição e Emagrecimento, 11(63), 135-144. http://www.rbone.com.br/index.php/rbone/article/view/504

Lacerda, M. S., Malheiros, G. C., \& Abreu, A. O. W. (2016). Tecido adiposo, uma nova visão: as adipocinas e seu papel endócrino / adiposo tissue, a newvision: adipocines and its endocrine paper. Revista Científica da Faculdade de Medicina de Campos, 11(2), 25-31. https://www.fmc.br/ojs/index.php/RCFMC/article/view/6

Liechocki, S. (2018). Avaliação do perfil inflamatório de diferentes depósitos de tecido adiposo na obesidade. Fiocruz.br, 1-128. https://www.arca.fiocruz.br/handle/icict/26991

Longo, M., Zatterale, F., Naderi, J., Parrillo, L., Formisano, P., Raciti, G. A., Beguinot, F., \& Miele, C. (2019). Adipose Tissue Dysfunction as Determinant of Obesity-Associated Metabolic Complications. International Journal of Molecular Sciences, 20(9), 2358. https://doi.org/10.3390/ijms20092358

Magdalon, J., \& Festuccia, W. T. (2017). Regulation of adiposity by mTORC1. Einstein (São Paulo), 15(4), 507-511. https://doi.org/10.1590/s1679$45082017 \mathrm{rb} 4106$

Magnuson, A. M., Fouts, J. K., Regan, D. P., Booth, A. D., Dow, S. W., \& Foster, M. T. (2018). Adipose tissue extrinsic factor: Obesity-induced inflammation and the role of the visceral lymph node. Physiology \& Behavior, 190, 71-81. https://doi.org/10.1016/j.physbeh.2018.02.044

Mendes, K. S, Silveira, R. C. C. P., \& Galvão, C. M. (2008). Revisão integrativa: método de pesquisa para a incorporação de evidências na saúde e na enfermagem. Texto contexto - enferm.,17, (4), 758-764. https://doi.org/10.1590/S0104-07072008000400018

Ministério da Saúde. (2019). Brasileiros atingem maior índice de obesidade dos últimos treze anos, de acordo com pesquisa Vigitel. Instituto nacional do câncer.

Nascimento, S. S. C., Queiroz, J. L. C., Medeiros, A. F., Nunes, A. C. F., Piuvezam, G. Maciel, B. L. L., Passos, T. S., \& Morais, A. H. A. (2021). What are the mechanisms of action of anti-inflammatory agentes in adipose tissue? Medicine, 100 (8), 24677. https://doi.org/10.1097/md.0000000000024677

Russo, L., \& Lumeng, C. N. (2018). Propriedades e funções dos macrófagos do tecido adiposo na obesidade. Immunology, 155 (4), $407-417$. https://doi.org/10.1111/imm.13002

Santos, C. M. C., Pimenta, C. A. M., \& Nobre, M. R. C. (2007) A estratégia PICO para a construção da pergunta de pesquisa e busca de evidências. Rev. Latino-Am. Enfermagem, 15 (3), 508-511. https://doi.org/10.1590/S0104-11692007000300023

Santos, F. M. M. (2017). Obesidade e estado inflamatório: principais adipocinas envolvidas. Universidade Federal do Rio Grande do Sul- LUME- Repositório Digital, 1-28.

Silva, N. I., Rocha Sobrinho, H. M., Blanch, G. T., Cruvinel, W. M., \& Gomes, M. G. (2019). Adipocinas e sua relação com a obesidade. Revista EVS Revista de Ciências Ambientais e Saúde, 46 (1), 53-64. https://doi.org/10.18224/evs.v46i1.7179 
Research, Society and Development, v. 10, n. 9, e45810918191, 2021

(CC BY 4.0) | ISSN 2525-3409 | DOI: http://dx.doi.org/10.33448/rsd-v10i9.18191

Soares, P. E. M., Ferreira, E. T. de O., Mafra, G. C. S., Silva, J. G. Santos, R. L. dos, Farias, J. V. C., \& Farias, I. C. C. (2020). O Papel das adipocinas na imunologia da obesidade. Research, Society and Development, 9 (7), e935975095. https://doi.org/10.33448/rsd-v9i7.5095

Suárez-Carmona, W., Sánchez-Oliver, A. J. \& González-Jurado, J. A. (2017) Fisiopatologia da obesidade: Perspectiva atual. Revista chilena de nutrição, 44 (3), 226-233. https://dx.doi.org/10.4067/s0717-75182017000300226

Veronese, A., Carmona, E. V., Silva, J. B., Carvalho, S. D., Trevisa, D. D., \& Beck, A. N. M. (2015). Revisão Sistemática ou Integrativa. REME - Revista Mineira de Enfermagem, 19 (3), 768-773. https://doi.org/10.5935/1415-2762.20150058 